\title{
The Extended Bloch Group and the Cheeger-Chern-Simons Class
}

\author{
Sebastian Goette \\ CHRISTIAN K ZICKERT
}

\begin{abstract}
We present a formula for the full Cheeger-Chern-Simons class of the tautological flat complex vector bundle of rank 2 over $B S L\left(2, \mathbb{C}^{\delta}\right)$. This improves the formula by Dupont and Zickert [6], where the class is only computed modulo 2-torsion.
\end{abstract}

57R20, 11G55

\section{Introduction}

The Cheeger-Chern-Simons class $\widehat{c}_{k}$ is a natural refinement of the $k$ th Chern class for complex vector bundles with connection, and it takes values in the ring of differential characters, see Chern-Simons [2] and Cheeger-Simons [1]. For a vector bundle with a flat connection, this class becomes an ordinary $(2 k-1)$-cohomology class with coefficients in $\mathbb{C} / \mathbb{Z}(k)$, where $\mathbb{Z}(k)=(2 \pi i)^{k} \mathbb{Z}$. Let $B S L\left(n, \mathbb{C}^{\delta}\right)$ denote the classifying space of the group $S L\left(n, \mathbb{C}^{\delta}\right)$ with the discrete topology. The universal Cheeger-ChernSimons class $\widehat{c}_{k} \in H^{2 k-1}\left(B S L\left(n, \mathbb{C}^{\delta}\right), \mathbb{C} / \mathbb{Z}(k)\right)$ of the tautological flat complex vector bundle over $B S L\left(n, \mathbb{C}^{\delta}\right)$ gives rise to the Borel regulator in algebraic $K$-theory, and $\widehat{c}_{2}$ is also related to invariants of hyperbolic 3-manifolds. One is interested in a combinatorial description of this class $\widehat{c}_{k} \in H^{2 k-1}\left(B S L\left(n, \mathbb{C}^{\delta}\right), \mathbb{C} / \mathbb{Z}(k)\right)$. Dupont derived an expression for $\widehat{c}_{2} \in H^{3}\left(B S L\left(2, \mathbb{C}^{\delta}\right), \mathbb{C} / \mathbb{Z}(2)\right)$ modulo $\mathbb{Q}(2)$ in [3]. A similar formula for $\operatorname{Re} \widehat{c}_{3}$ is due to Goncharov [7].

The homology of the classifying space of a discrete group is by definition the homology of the group, and since $\mathbb{C} / \mathbb{Z}(2)$ is divisible, we can regard $\widehat{c}_{2}$ as a homomorphism $H_{3}(S L(2, \mathbb{C})) \rightarrow \mathbb{C} / \mathbb{Z}(2)$. The natural map $H_{3}(S L(2, \mathbb{C})) \rightarrow H_{3}(P S L(2, \mathbb{C}))$ has cyclic kernel of order 4 , so we have a commutative diagram defining $\widehat{c}_{2}$ on $H_{3}(P S L(2, \mathbb{C}))$ :

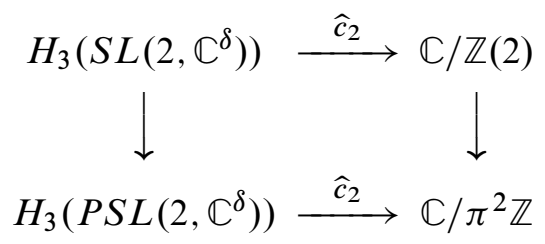


An explicit formula for the lower map was obtained by Neumann [8], and extended by Dupont and Zickert [6] to a formula for the upper map. However, the formula given in [6] only computes the image of $\widehat{c}_{2}$ in $\mathbb{C} / 2 \pi^{2} \mathbb{Z}$, thus only computing $\widehat{c}_{2}$ up to 2 -torsion (see [6, Remark 4.2] for a comment on the normalisation). In the present paper we extend their result obtaining a formula computing the full Cheeger-Chern-Simons class.

In both [8] and [6] the formulas are obtained by factoring $\widehat{c}_{2}$ through a version of the extended Bloch group, an object defined by Neumann in [8]. There are two different versions of the extended Bloch group. One version, denoted $\widehat{\mathcal{B}}(\mathbb{C})$ in Neumann's paper and $\widehat{\mathcal{B}}_{P S L}(\mathbb{C})$ later on in this note, is generated by symbols $[z ; p, q]$ subject to a five term relation and a transfer relation. It is isomorphic to $H_{3}\left(P S L\left(2, \mathbb{C}^{\delta}\right)\right)$. The other one, denoted $\mathcal{E} \mathcal{B}(\mathbb{C})$ in $[8]$ and $\widehat{\mathcal{B}}_{S L}(\mathbb{C})$ in the following, is generated by symbols $[z ; 2 p, 2 q]$ and only subject to the five term relation. The latter version is called the more extended Bloch group, and conjectured to be isomorphic to $H_{3}\left(S L\left(2, \mathbb{C}^{\delta}\right)\right)$ in [8]. The role of the transfer relation is subtle and has caused some minor inaccuracies in [8] and [6], see Remark 3.3 below. [8, Proposition 8.2 and Corollary 8.3] are only correct if we include the transfer relation. Proposition 8.2 has been used in the proof of [6, Proposition 4.15], so this result is also only correct if we include the transfer relation. To the best of our knowledge these are the only problems in [8] and [6]. We present corrections to these results as Theorem 3.4, Corollary 3.5 and Corollary 4.2, and Remark 4.3 below. This paper can thus be viewed as an erratum and a refinement of the papers [8] and [6].

To obtain the full class $\widehat{c}_{2}$, we construct a lift of the function $\widehat{L}: \widehat{\mathcal{B}}_{S L}(\mathbb{C}) \rightarrow \mathbb{C} / 2 \pi^{2} \mathbb{Z}$ defined by Dupont and Zickert [6] with values in $\mathbb{C} / 4 \pi^{2} \mathbb{Z}=\mathbb{C} / \mathbb{Z}(2)$. Note that this lift is not compatible with the transfer relation, see Remark 3.3. This observation was the main motivation for the present note.

The paper is organised as follows: In Section 1 we recall the definition of the extended Bloch group. In Section 2 we define the modified extended Rogers dilogarithm. In Section 3 we work out some relations in the extended Bloch group, and in Section 4 we prove our main results that the extended Bloch group is isomorphic to $H_{3}\left(S L\left(2, \mathbb{C}^{\delta}\right)\right)$ as conjectured by Neumann [8], and that $\widehat{L}$ computes the Cheeger-Chern-Simons class. In Section 5, we have added some more relations in $\widehat{\mathcal{P}}(\mathbb{C})$ that might be of interest elsewhere.

\section{Acknowledgements}

We are grateful to J Dupont, G Kings and W Neumann for fruitful discussions and their interest in our work. The first named author was supported in part by DFG special 
programme "Global Differential Geometry"; parts of this note were written while the first named author enjoyed the hospitality of the Chern Institute at Tianjin. The second named author was supported by "Rejselegat for matematikere".

\section{The extended Bloch group}

We follow Neumann's description in [8] at the beginning of chapter 8. We first recall the construction of the classical Bloch group. Define a set of five term relations

$$
\mathrm{FT}=\left\{\left(x, y, \frac{y}{x}, \frac{1-1 / x}{1-1 / y}, \frac{1-x}{1-y}\right) \mid x \neq y \in \mathbb{C} \backslash\{0,1\}\right\} \subset(\mathbb{C} \backslash\{0,1\})^{5} .
$$

Consider the free Abelian groups generated by the elements of FT and $\mathbb{C} \backslash\{0,1\}$ and the chain complex

$$
\mathbb{Z}[\mathrm{FT}] \stackrel{\rho}{\longrightarrow} \mathbb{Z}[\mathbb{C} \backslash\{0,1\}] \stackrel{v}{\longrightarrow} \mathbb{C}^{\times} \wedge \mathbb{Z} \mathbb{C}^{\times}
$$

with arrows defined on generators by

$$
\begin{aligned}
\rho\left(\left[z_{0}, \ldots, z_{4}\right]\right) & =\left[z_{0}\right]-\left[z_{1}\right]+\left[z_{2}\right]-\left[z_{3}\right]+\left[z_{4}\right], \\
v([z]) & =z \wedge(1-z)^{-1} .
\end{aligned}
$$

Then $\mathcal{P}(\mathbb{C})=\mathbb{Z}[\mathbb{C} \backslash\{0,1\}] /$ im $\rho$ is called the pre-Bloch group, and the middle homology of the complex (2) above is called the Bloch group $\mathcal{B}(\mathbb{C})$.

Let $\widehat{\mathbb{C}}$ denote the universal Abelian cover of $\mathbb{C} \backslash\{0,1\}$. To construct $\widehat{\mathbb{C}}$, we start with $\mathbb{C}_{\text {cut }}=\mathbb{C} \backslash((-\infty, 0] \cup[1, \infty))$. For each $x \in(-\infty, 0) \cup(1, \infty)$, we add two boundary points

$$
x \pm 0 i:=\lim _{t \searrow 0} x \pm t i
$$

and put

$$
\overline{\mathbb{C}}_{\text {cut }}=\mathbb{C}_{\text {cut }} \cup\{x \pm 0 i \mid x \in(-\infty, 0) \cup(1, \infty)\} .
$$

We extend the principal branches of $\log , \operatorname{Li}_{2}$ and $\mathrm{Arg}=\operatorname{Im} \log$ to $\overline{\mathbb{C}}_{\text {cut }}$.

In $\overline{\mathbb{C}}_{\text {cut }} \times(2 \mathbb{Z})^{2}$, identify

$$
\begin{aligned}
& (x+0 i, 2 p, 2 q) \sim(x-0 i, 2 p+2,2 q) \text { for all } x \in(-\infty, 0), \text { and } \\
& (x+0 i, 2 p, 2 q) \sim(x-0 i, 2 p, 2 q+2) \text { for all } x \in(1, \infty)
\end{aligned}
$$

for all $p, q \in \mathbb{Z}$, obtaining $\widehat{\pi}: \widehat{\mathbb{C}} \rightarrow \mathbb{C} \backslash\{0,1\}$. Equivalence classes will be denoted $(x ; 2 p, 2 q)$ or simply $\widehat{x}$. Note that we could have dropped the factors 2 above and worked in $\overline{\mathbb{C}}_{\text {cut }} \times \mathbb{Z}^{2}$ instead, but we want to stay compatible with [6] and [8]. 
As in [8], put

$$
\mathrm{FT}^{+}=\left\{\left(z_{0}, \ldots, z_{4}\right) \in \mathrm{FT} \mid \operatorname{Im} z_{0}, \ldots, \operatorname{Im} z_{4}>0\right\} .
$$

Then $\left(z_{0}, \ldots, z_{4}\right) \in$ FT belongs to $\mathrm{FT}^{+}$if and only if $\operatorname{Im} z_{1}>0$ and $z_{0}$ is in the interior of the Euclidean triangle spanned by 0,1 and $z_{1}$. Let $\widehat{\mathrm{FT}}$ denote the connected component of $\widehat{\pi}^{-1}(\mathrm{FT}) \subset \widehat{\mathbb{C}}^{5}$ that contains

$$
\widehat{\mathrm{FT}}^{+}=\left\{\left(\left(z_{0} ; 0,0\right), \ldots,\left(z_{4} ; 0,0\right)\right) \mid\left(z_{0}, \ldots, z_{4}\right) \in \mathrm{FT}^{+}\right\} .
$$

Also, note that the functions

$$
(z ; 2 p, 2 q) \longmapsto(\log z+2 \pi i p) \text { and }(z ; 2 p, 2 q) \longmapsto(-\log (1-z)+2 \pi i q)
$$

are holomorphic on $\widehat{\mathbb{C}}$. By [6], we can extend (2) to a chain complex

$$
\mathbb{Z}[\widehat{\mathrm{FT}}] \stackrel{\widehat{\rho}}{\longrightarrow} \mathbb{Z}[\widehat{\mathbb{C}}] \stackrel{\widehat{v}}{\longrightarrow} \mathbb{C} \wedge \mathbb{Z} \mathbb{C}
$$

with arrows defined on generators by

$$
\begin{aligned}
\widehat{\rho}\left(\left[\widehat{z}_{0}, \ldots, \widehat{z}_{4}\right]\right) & =\left[\widehat{z}_{0}\right]-\left[\widehat{z}_{1}\right]+\left[\widehat{z}_{2}\right]-\left[\widehat{z}_{3}\right]+\left[\widehat{z}_{4}\right], \\
\widehat{v}([z ; 2 p, 2 q]) & =(\log z+2 \pi i p) \wedge(-\log (1-z)+2 \pi i q) .
\end{aligned}
$$

1.1 Definition The extended pre-Bloch group $\widehat{\mathcal{P}}_{S L}(\mathbb{C})$ is defined as the quotient $\mathbb{Z}[\widehat{\mathbb{C}}] /$ im $\widehat{\rho}$, and the extended Bloch group $\widehat{\mathcal{B}}_{S L}(\mathbb{C})$ as the middle homology of the complex (4).

\section{The extended Rogers dilogarithm}

The classical dilogarithm is given by

$$
\operatorname{Li}_{2}(z)=\sum_{k=1}^{\infty} \frac{z^{k}}{k^{2}}=-\int_{0}^{z} \log (1-t) \frac{d t}{t}
$$

for all $z$ with $|z|<1$. It extends to a multivalued function on $\mathbb{C}$ with branch points at 0,1 and $\infty$. Recall that the Rogers dilogarithm $L:(0,1) \rightarrow \mathbb{R}$ is given by

$$
L(x)=\operatorname{Li}_{2}(x)+\frac{1}{2} \log x \log (1-x)-\frac{\pi^{2}}{6} .
$$

We extend $L$ to a holomorphic function:

$$
\begin{gathered}
\bar{L}: \overline{\mathbb{C}}_{\text {cut }} \times(2 \mathbb{Z})^{2} \longrightarrow \mathbb{C} \\
\bar{L}(z ; 2 p, 2 q)=\operatorname{Li}_{2}(z)+\frac{1}{2}(\log z+2 \pi i p)(\log (1-z)+2 \pi i q)-\frac{\pi^{2}}{6} .
\end{gathered}
$$


2.1 Lemma The function $\bar{L}$ above induces a holomorphic function

$$
\widehat{L}: \widehat{\mathbb{C}} \rightarrow \mathbb{C} / \mathbb{Z}(2)
$$

that satisfies the five term relation

$$
\sum_{k=0}^{4}(-1)^{k} \widehat{L}\left(\widehat{z}_{k}\right)=0
$$

for all $\left(\widehat{z}_{0}, \ldots, \widehat{z}_{4}\right) \in \widehat{\mathrm{FT}}$.

Note that $\widehat{L}$ lifts Dupont and Zickert's function $\widehat{L}$ in [6] from $\mathbb{C} / 2 \pi^{2} \mathbb{Z}$ to $\mathbb{C} / 4 \pi^{2} \mathbb{Z}=$ $\mathbb{C} / \mathbb{Z}(2)$.

Proof Because $\operatorname{Li}_{2}(z)$ has no singularity at $z=0$ and $(z ; p, q) \mapsto \log z+2 \pi i p$ is holomorphic on $\widehat{\mathbb{C}}$, the function $\bar{L}$ extends holomorphically across $(-\infty, 0) \times(2 \mathbb{Z})^{2}$ in $\widehat{\mathbb{C}}$.

If we extend $\operatorname{Li}_{2}$ and $\log (1-z)$ to $z \pm 0 i$ for $z>1$, then

$$
\begin{aligned}
\operatorname{Li}_{2}(z+0 i) & =\operatorname{Li}_{2}(z-0 i)+2 \pi i \log z \\
\log (1-(z+0 i)) & =\log (1-(z-0 i))-2 \pi i .
\end{aligned}
$$

Hence

$$
\bar{L}(z+0 i ; 2 p, 2 q)=\bar{L}(z-0 i ; 2 p, 2 q+2)+4 \pi^{2} p,
$$

so the extension $\widehat{L}$ of $\bar{L} \bmod 4 \pi^{2}$ is well-defined.

By Neumann [8], we have a five term relation

$$
\sum_{k=0}^{4}(-1)^{k} \bar{L}\left(\widehat{z}_{k}\right)=0 \in \mathbb{C}
$$

for all $\left(\widehat{z}_{0}, \ldots, \widehat{z}_{4}\right) \in \widehat{\mathrm{FT}}^{+}$. Because $\widehat{\mathrm{FT}}$ is a connected complex manifold, the five term relation for $\widehat{L}$ holds in $\mathbb{C} / \mathbb{Z}(2)$ for all $\left(\widehat{z}_{0}, \ldots, \widehat{z}_{4}\right) \in \widehat{\mathrm{FT}}$ by analytic continuation.

2.2 Remark Along the commutator of a small loop around 0 and a small loop around 1 in $\mathbb{C} \backslash\{0,1\}$, the holomorphic continuation of the Rogers dilogarithm changes by $4 \pi^{2}$. This shows that we cannot lift $L$ to a holomorphic function on $\widehat{\mathbb{C}}$ with values in $\mathbb{C} / A$, with $A \subset \mathbb{Z}(2)$ a proper subgroup.

2.3 Corollary The function $\widehat{L}$ induces homomorphisms

$$
\widehat{L}: \widehat{\mathcal{P}}_{S L}(\mathbb{C}) \rightarrow \mathbb{C} / \mathbb{Z}(2) \text { and } \widehat{L}: \widehat{\mathcal{B}}_{S L}(\mathbb{C}) \rightarrow \mathbb{C} / \mathbb{Z}(2) \text {. }
$$




\section{Relations in the extended Bloch group}

Following Neumann [8], we find relations among elements of the extended pre-Bloch group. We then parameterise the kernel of the forgetful maps $\widehat{\mathcal{P}}_{S L}(\mathbb{C}) \rightarrow \mathcal{P}(\mathbb{C})$ and $\widehat{\mathcal{B}}_{S L}(\mathbb{C}) \rightarrow \mathcal{B}(\mathbb{C})$ induced by the projection $\widehat{\pi}: \widehat{\mathbb{C}} \rightarrow \mathbb{C}$. In the following, we will identify $z \in(-\infty, 0) \cup(1, \infty)$ with $z+0 i$.

As explained by Dupont and Zickert in the appendix of [6]:

$$
\begin{aligned}
& \widehat{\pi}^{-1}\left(\mathrm{FT}^{+}\right) \cap \widehat{\mathrm{FT}}=\left\{\left(\left(z_{0} ; 2 p_{0}, 2 q_{0}\right),\left(z_{1} ; 2 p_{1}, 2 q_{1}\right),\left(z_{2} ; 2\left(p_{1}-p_{0}\right), 2 q_{2}\right),\right.\right. \\
& \left.\left(z_{3} ; 2\left(p_{1}-p_{0}+q_{1}-q_{0}\right), 2\left(q_{2}-q_{1}\right)\right),\left(z_{4} ; 2\left(q_{1}-q_{0}\right), 2\left(q_{2}-q_{1}-p_{0}\right)\right)\right) \\
& \left.\mid\left(z_{0}, \ldots, z_{4}\right) \in \mathrm{FT}^{+} \text {and } p_{0}, p_{1}, q_{0}, q_{1}, q_{2} \in \mathbb{Z}\right\} .
\end{aligned}
$$

For other choices of $\left(z_{0}, \ldots, z_{4}\right) \in \mathrm{FT}$, a few of the $p_{k}, q_{k}$ have to be adjusted by \pm 1 .

Subtracting two instances of the five term relation and using (1) and (6), we obtain Neumann's cycle relation

$$
\begin{aligned}
{\left[x ; 2 p_{0}, 2 q_{0}-2\right]-\left[y ; 2 p_{1}, 2 q_{1}-2\right]+\left[\frac{y}{x} ; 2 p_{1}-2 p_{0}, 2 q_{2}-2\right] } & \\
= & {\left[x ; 2 p_{0}, 2 q_{0}\right]-\left[y ; 2 p_{1}, 2 q_{1}\right]+\left[\frac{y}{x} ; 2 p_{1}-2 p_{0}, 2 q_{2}\right], }
\end{aligned}
$$

for all $x, y$ such that $\left(x, y, \frac{y}{x}, \frac{1-1 / x}{1-1 / y}, \frac{1-x}{1-y}\right) \in \mathrm{FT}^{+}$. If we vary $x$ and $y$ continuously, then some of the integers in this relation may jump. Thus, we obtain

$$
\begin{aligned}
{\left[x ; 2 p_{0}, 2 q_{0}-2\right]-\left[x ; 2 p_{0}, 2 q_{0}\right]-\left[y ; 2 p_{1}, 2 q_{1}-2\right]+\left[y ; 2 p_{1}, 2 q_{1}\right] } \\
\quad=\left\{\begin{array}{cl}
{\left[\frac{y}{x} ; 2 p_{1}-2 p_{0}-2,2 q_{2}-2\right]} & \begin{array}{c}
{\left[\frac{y}{x} ; 2 p_{1}-2 p_{0}, 2 q_{2}-2\right]} \\
-\left[\frac{y}{x} ; 2 p_{1}-2 p_{0}-2,2 q_{2}\right]
\end{array} \\
-\left[\frac{y}{x} ; 2 p_{1}-2 p_{0}, 2 q_{2}\right] & \text { if } \operatorname{Arg} y-\operatorname{Arg} x \leq-\pi, \\
{\left[\frac{y}{x} ; 2 p_{1}-2 p_{0}+2,2 q_{2}-2\right]} & \text { if }-\pi<\operatorname{Arg} x \leq \pi, \\
-\left[\frac{y}{x} ; 2 p_{1}-2 p_{0}+2,2 q_{2}\right] & \text { if } \pi<\operatorname{Arg} y-\operatorname{Arg} x .
\end{array}\right.
\end{aligned}
$$

Subtracting two instances of (7) gives

$$
[x ; 2 p, 2(q-1)]-[x ; 2 p, 2 q]=\left[x ; 2 p, 2\left(q^{\prime}-1\right)\right]-\left[x ; 2 p, 2 q^{\prime}\right]
$$


for all $x \in \overline{\mathbb{C}}_{\text {cut }}$. Similarly, one can prove

$$
[x ; 2(p-1), 2 q]-[x ; 2 p, 2 q]=\left[x ; 2\left(p^{\prime}-1\right), 2 q\right]-\left[x ; 2 p^{\prime}, 2 q\right]
$$

and

$$
[x ; 2(p+1), 2(q-1)]-[x ; 2 p, 2 q]=\left[x ; 2\left(p^{\prime}+1\right), 2\left(q^{\prime}-1\right)\right]-\left[x ; 2 p^{\prime}, 2 q^{\prime}\right]
$$

for all $x \in \overline{\mathbb{C}}_{\text {cut }}$ and all $p, q, p^{\prime}, q^{\prime} \in \mathbb{Z}$ such that $p^{\prime}+q^{\prime}=p+q$. See Neumann [8] for a more geometric derivation of these relations.

By [8, Lemma 7.3], we also have the relation

$$
[z ; 2 p, 2 q]+[1-z ;-2 q,-2 p]=2\left[\frac{1}{2} ; 0,0\right],
$$

which is of course compatible with (8)-(10).

Let us define

$$
\{z ; 2 p\}=[z ; 2 p, 2 q]-[z ; 2 p, 2(q-1)],
$$

which is independent of $q$ by (8).

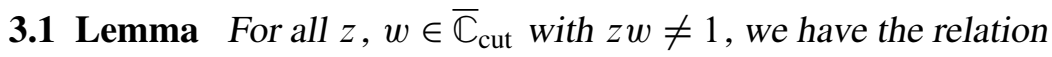

$$
\{z ; 2 p\}+\{w ; 2 r\}= \begin{cases}\{z w+0 i ; 2(p+r-1)\} & \text { if } \operatorname{Arg} z+\operatorname{Arg} w \leq-\pi, \\ \{z w+0 i ; 2(p+r)\} & \text { if }-\pi<\operatorname{Arg} z+\operatorname{Arg} w \leq \pi \text { and } \\ \{z w+0 i ; 2(p+r+1)\} & \text { if } \pi<\operatorname{Arg} z+\operatorname{Arg} w .\end{cases}
$$

Proof This is immediate from (7).

3.2 Lemma The element $\widehat{\kappa}=\{z ; 2 p\}-\{z ; 2(p-1)\} \in \widehat{\mathcal{P}}_{S L}(\mathbb{C})$ is independent of $z \in \mathbb{C} \backslash\{0,1\}$ and $p \in \mathbb{Z}$, and of order 2 in $\widehat{\mathcal{P}}_{S L}(\mathbb{C})$.

Proof Independence of $p$ follows from (9) and independence of $z$ is immediate from Lemma 3.1. To prove that $\widehat{\kappa}$ is of order two, use (8) and (11) to write

$$
\begin{aligned}
\widehat{\kappa} & =[z ; 2,0]-[z ; 2,-2]-[z ; 0,0]+[z ; 0,-2] \\
& =-[1-z ; 0,-2]+[1-z ; 2,-2]+[1-z ; 0,0]-[1-z ; 2,0] \\
& =\{1-z ; 0\}-\{1-z ; 2\}=-\widehat{\kappa} .
\end{aligned}
$$

To show that $\widehat{\kappa} \neq 0$, we compute

$$
\widehat{L}(\widehat{\kappa})=\widehat{L}(z ; 2,2)-\widehat{L}(z ; 2,0)-\widehat{L}(z ; 0,2)+\widehat{L}(z ; 0,0)=-2 \pi^{2},
$$

and $-2 \pi^{2} \neq 0$ in $\mathbb{C} / \mathbb{Z}(2)$. 
3.3 Remark Neumann has implicitly assumed that $\widehat{\kappa}=0$ in [8, Section 8], and his conclusions have then been used by Dupont and Zickert in [6, Proposition 4.15]. More precisely, Neumann introduces a "transfer relation"

$$
[z ; p, q]+\left[z ; p^{\prime}, q^{\prime}\right]=\left[z ; p, q^{\prime}\right]+\left[z ; p, q^{\prime}\right] \text { for all } p, q, p^{\prime}, q^{\prime} \in \mathbb{Z}
$$

in the definition of his extended Bloch group. The analogous relation in our context would read

$$
[z ; 2 p, 2 q]+\left[z ; 2 p^{\prime}, 2 q^{\prime}\right]=\left[z ; 2 p, 2 q^{\prime}\right]+\left[z ; 2 p, 2 q^{\prime}\right] \text { for all } p, q, p^{\prime}, q^{\prime} \in \mathbb{Z} .
$$

In analogy with [8, Proposition 7.2], one can show that the effect of the transfer relation above is equivalent to dividing by the subgroup of order two that is generated by $\widehat{\kappa}$.

We have just computed $\widehat{L}(\widehat{\kappa})=-2 \pi^{2} \in \mathbb{C} / 4 \pi^{2} \mathbb{Z}$ in (12). This explains that if one includes the transfer relation, then $\widehat{L}$ is well-defined only modulo $2 \pi^{2} \mathbb{Z}$ as in [6].

Assuming that $\widehat{\kappa}=0$, one finds that $\{z ; 2 p\}$ becomes independent of $p$. This allows the definition of a homomorphism $\mathbb{C}^{\times} \rightarrow \widehat{\mathcal{P}}_{S L}(\mathbb{C}) /\langle\widehat{\kappa}\rangle$ with $z \mapsto\{z ; 0\}$, see [8, Proposition 8.2].

Starting from Neumann's map, we obtain a pullback square

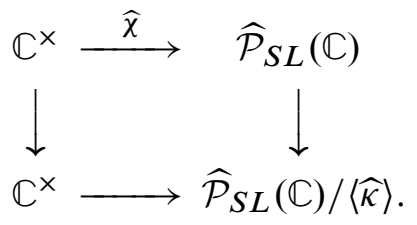

Here the left vertical arrow maps $z$ to $z^{2}$, and $\widehat{\chi}$ is given by

$$
\widehat{\chi}(z)= \begin{cases}0 & \text { if } z=1, \\ \widehat{\kappa} & \text { if } z=-1, \\ \left\{z^{2}+0 i ; 0\right\} & \text { if } \operatorname{Arg} z \in\left(-\frac{\pi}{2}, \frac{\pi}{2}\right] \text { and } z \neq 1, \text { and } \\ \left\{z^{2}+0 i ; 2\right\} & \text { if } \operatorname{Arg} z \notin\left(-\frac{\pi}{2}, \frac{\pi}{2}\right] \text { and } z \neq-1 .\end{cases}
$$

3.4 Theorem The map $\widehat{\chi}$ is a homomorphism, and the sequence

$$
0 \longrightarrow \mathbb{C}^{\times} \stackrel{\widehat{\chi}}{\longrightarrow} \widehat{\mathcal{P}}_{S L}(\mathbb{C}) \longrightarrow \mathcal{P}(\mathbb{C}) \longrightarrow 0
$$

is exact and split, where the right arrow is induced by $\widehat{\pi}: \widehat{\mathbb{C}} \rightarrow \mathbb{C} \backslash\{0,1\}$.

Proof First of all we note that by the definition of $\widehat{\kappa}$ and Lemma 3.2,

$$
\left\{z^{2} ; 2 p\right\}+\widehat{\kappa}=\left\{z^{2} ; 2 p+2\right\}=\left\{z^{2} ; 2 p-2\right\},
$$


which implies that $\widehat{\chi}(z)+\widehat{\chi}(-1)=\widehat{\chi}(-z)$, and that $\left\{z^{2}, 2 p+4\right\}=\left\{z^{2} ; 2 p\right\}$. By Lemma 3.1, we have $\widehat{\chi}(z)+\widehat{\chi}(w)=\widehat{\chi}(z w)$ for almost all choices of $z, w \in \mathbb{C}^{\times}$. The remaining cases are easily checked.

The right arrow maps $\{z ; 2 p\}=[z ; 2 p, 2]-[z ; 2 p, 0]$ to $[z]-[z]=0$, so the sequence above is a chain complex. To prove injectivity of $\widehat{\chi}$ consider the composition $\widehat{L} \circ \widehat{\chi}$. For $z \in \mathbb{C}^{\times}$let $p=0$ if $\operatorname{Arg} z \in\left(-\frac{\pi}{2}, \frac{\pi}{2}\right]$ and $p=1$ if $\operatorname{Arg} z \notin\left(-\frac{\pi}{2}, \frac{\pi}{2}\right]$. Then

$$
\log \left(z^{2}+0 i\right)+2 \pi i p \equiv 2 \log z \text { modulo } 2 \pi i \mathbb{Z},
$$

and

$$
\begin{aligned}
(\widehat{L} \circ \widehat{\chi})(z)=\frac{1}{2}\left(\left(\log z^{2}+2 \pi i p\right)\left(\log \left(1-z^{2}\right)+2 \pi i\right)\right. & \\
\left.-\left(\log z^{2}+2 \pi i p\right) \log \left(1-z^{2}\right)\right) & =2 \pi i \log z \in \mathbb{C} / \mathbb{Z}(2),
\end{aligned}
$$

and this even holds for $z= \pm 1$ by (12), (13). Hence $\widehat{\chi}$ is injective.

It remains to show that $\operatorname{im} \widehat{\chi}=\operatorname{ker}\left(\widehat{\mathcal{P}}_{S L}(\mathbb{C}) \rightarrow \mathcal{P}(\mathbb{C})\right)$. Relations (8) and (9) allow us to represent each generator of $\widehat{\mathcal{P}}_{S L}(\mathbb{C})$ as

$[z ; 2 p, 2 q]=p q[z ; 2,2]-p(q-1)[z ; 2,0]-(p-1) q[z ; 0,2]+(p-1)(q-1)[z ; 0,0]$,

see $\left[8\right.$, Lemma 7.1]. Using (11), we see that the kernel of $\widehat{\mathcal{P}}_{S L}(\mathbb{C}) \rightarrow \mathcal{P}(\mathbb{C})$ is generated by elements of the form

$$
[z ; 2 p, 2 q]-[z ; 0,0]=p q\{z ; 2\}-(p-1) q\{z ; 0\}+p\{1-z ; 0\} \in \operatorname{im} \widehat{\chi} .
$$

By (14), a splitting of the sequence is given by the homomorphism

$$
\exp \circ \frac{\widehat{L}}{2 \pi i}: \widehat{\mathcal{P}}_{S L}(\mathbb{C}) \rightarrow \mathbb{C}^{\times}
$$

3.5 Corollary cf Neumann [8, Theorem 7.5] The sequence

$$
0 \longrightarrow \mathbb{Q} / \mathbb{Z} \stackrel{\alpha \mapsto \widehat{\chi}\left(e^{2 \pi i \alpha}\right)}{\longrightarrow} \widehat{\mathcal{B}}_{S L}(\mathbb{C}) \longrightarrow \mathcal{B}(\mathbb{C}) \longrightarrow 0
$$

is exact and

$$
\frac{1}{(2 \pi i)^{2}} \widehat{L}\left(\widehat{\chi}\left(e^{2 \pi i \alpha}\right)\right)=\alpha .
$$




\section{The Cheeger-Chern-Simons class and $H_{3}\left(S L\left(2, \mathbb{C}^{\delta}\right)\right)$}

Recall that Dupont and Zickert have constructed a map $\widehat{\lambda}: H_{3}\left(S L\left(2, \mathbb{C}^{\delta}\right)\right) \rightarrow \widehat{\mathcal{B}}_{S L}(\mathbb{C})$ in [6, Section 3] without using the transfer relation. Following [6], we prove that $\widehat{L} \circ \widehat{\lambda}=\widehat{c}_{2} \in \mathbb{C} / \mathbb{Z}(2)$ and conclude from this that $\widehat{\lambda}$ is an isomorphism.

Note that because $\mathbb{C} / \mathbb{Z}(2)$ is divisible, there is a canonical isomorphism

$$
H^{3}\left(S L\left(2, \mathbb{C}^{\delta}\right), \mathbb{C} / \mathbb{Z}(2)\right) \cong \operatorname{Hom}_{\mathbb{Z}}\left(H_{3}\left(S L\left(2, \mathbb{C}^{\delta}\right)\right), \mathbb{C} / \mathbb{Z}(2)\right) .
$$

Let $\widehat{c}_{2} \in H^{3}\left(S L\left(2, \mathbb{C}^{\delta}\right), \mathbb{C} / \mathbb{Z}(2)\right)$ denote the second Cheeger-Chern-Simons class of the tautological flat complex vector bundle of rank 2 over $B S L\left(2, \mathbb{C}^{\delta}\right)$. Here, we are using the same normalisation as Neumann [8]. Dupont and Zickert consider the class $\frac{1}{(2 \pi i)^{2}} \widehat{c}_{2} \in H^{3}\left(S L\left(2, \mathbb{C}^{\delta}\right), \mathbb{C} / \mathbb{Z}\right)$, see [6, Remark 4.2].

4.1 Theorem cf Dupont and Zickert [6, Theorem 4.1] Under the isomorphism above,

$$
\widehat{c}_{2}=\widehat{L} \circ \widehat{\lambda}
$$

Proof By [6, Theorem 4.1], we have that

$$
2 \widehat{c}_{2}=2 \widehat{L} \circ \widehat{\lambda} \in \operatorname{Hom}_{\mathbb{Z}}\left(H_{3}\left(S L\left(2, \mathbb{C}^{\delta}\right)\right), \mathbb{C} / \mathbb{Z}(2)\right)
$$

in our normalisation. Because $H_{3}\left(S L\left(2, \mathbb{C}^{\delta}\right)\right)$ is divisible, this implies our claim.

4.2 Corollary $\operatorname{cf}\left[6\right.$, Theorem 4.15] The map $\widehat{\lambda}: H_{3}\left(S L\left(2, \mathbb{C}^{\delta}\right)\right) \rightarrow \widehat{\mathcal{B}}_{S L}(\mathbb{C})$ is an isomorphism.

Proof Consider the commutative diagram

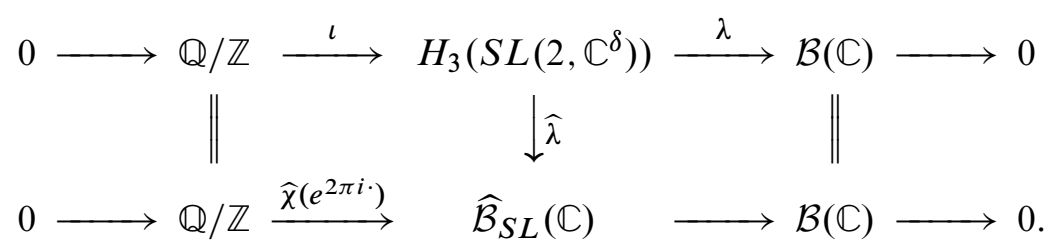

Exactness of the upper row has been established by Dupont and Sah in [5]. The lower row is just Corollary 3.5. Commutativity of the right hand square has been established in $[6$, Section 3].

Let $\alpha \in \mathbb{Q}$, then $\frac{1}{(2 \pi i)^{2}}\left(\widehat{c}_{2} \circ \iota\right)(\alpha)=\alpha$ by Dupont [4, Theorem 10.2]. Theorem 4.1 implies that

$$
\frac{1}{(2 \pi i)^{2}} \widehat{L}((\lambda \circ \iota)(\alpha))=\frac{1}{(2 \pi i)^{2}}\left(\widehat{c}_{2} \circ \iota\right)(\alpha)=\alpha,
$$


and $(\lambda \circ \iota)(\alpha) \in \operatorname{ker}\left(\widehat{\mathcal{B}}_{S L}(\mathbb{C}) \rightarrow \mathcal{B}(\mathbb{C})\right)$ by commutativity of the right hand square. On the other hand, Corollary 3.5 implies that

$$
\frac{1}{(2 \pi i)^{2}} \widehat{L}\left(\widehat{\chi}\left(e^{2 \pi i \alpha}\right)\right)=\alpha,
$$

and that $\widehat{L}$ is injective on $\operatorname{ker}\left(\widehat{\mathcal{B}}_{S L}(\mathbb{C}) \rightarrow \mathcal{B}(\mathbb{C})\right)$. Thus the left hand square also commutes. Our claim now follows from the five-lemma.

4.3 Remark Let $\widehat{\mathcal{B}}_{P S L}(\mathbb{C}) \cong H_{3}(P S L(2, \mathbb{C}))$ denote Neumann's extended Bloch group in [8]. Then the diagram

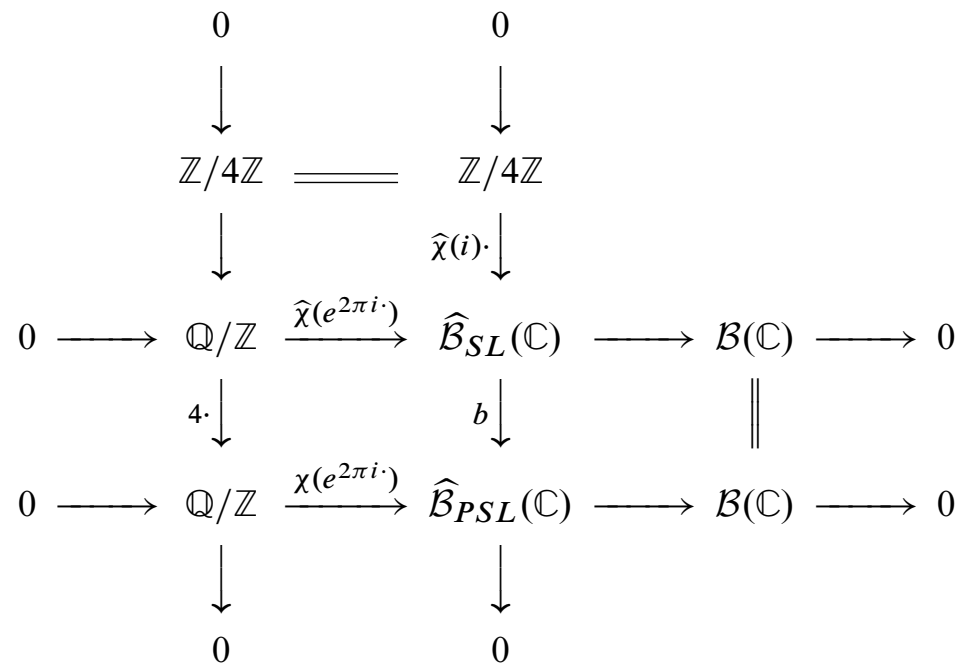

commutes and has exact rows and columns. Here the map $b: \widehat{\mathcal{B}}_{S L}(\mathbb{C}) \rightarrow \widehat{\mathcal{B}}_{P S L}(\mathbb{C})$ sends a generator $[z, 2 p, 2 q]$ to the same generator in $\widehat{\mathcal{B}}_{P S L}(\mathbb{C})$, and $\chi$ has been defined in [8, Proposition 7.4]. This is proved in analogy with in [8, Corollary 8.3]. For example, commutativity of the lower left hand square follows from

$$
\begin{aligned}
(b \circ \widehat{\chi})(z) & =\left[z^{2} ; 2 p, 2\right]-\left[z^{2} ; 2 p, 0\right] \\
& =2\left(\left[z^{2} ; 2 p, 1\right]-\left[z^{2} ; 2 p, 0\right]\right)=2 \chi\left(z^{2}\right)=4 \chi(z) \in \widehat{\mathcal{P}}_{P S L}(\mathbb{C}) .
\end{aligned}
$$

This also shows that $\operatorname{ker} b$ is spanned by $\widehat{\chi}(i)=\{-1 ; 0\}$.

\section{More relations in the extended pre-Bloch group}

By Dupont and Sah [5], one has the relations

$$
[z]=\left[\frac{1}{1-z}\right]=\left[1-\frac{1}{z}\right]=-\left[\frac{1}{z}\right]=-[1-z]=-\left[-\frac{z}{1-z}\right]
$$


in the pre-Bloch group $\mathcal{P}(\mathbb{C})$. If we interpret $z$ as the cross-ratio of a generic configuration of four points in $\mathbb{C} P^{1}$, then these relations say that up to orientation, the order of the points is not important. Note that $\operatorname{Im} \widehat{c}_{2}$ is already well-defined on $\mathcal{B}(\mathbb{C})$.

Similar relations hold in Neumann's extended pre-Bloch group $\widehat{\mathcal{P}}_{P S L}(\mathbb{C})$ by $[8$, Proposition 13.1]. As a consequence, unordered oriented simplices are also sufficient to compute $\operatorname{Re} \widehat{c}_{2}$ up to some finite ambiguity. Unfortunately, these relations become more complicated in $\widehat{\mathcal{P}}_{S L}(\mathbb{C})$. Let $\sqrt[4]{z}$ denote the standard fourth root of $z$ with $\operatorname{Arg} \sqrt[4]{z} \in\left(-\frac{\pi}{4}, \frac{\pi}{4}\right]$.

\subsection{Proposition Let $\operatorname{Im} z>0$. Then}

$$
\begin{aligned}
{\left[\frac{1}{z} ;-2 p, 2 p+2 q\right] } & =-[z ; 2 p, 2 q]+\widehat{\chi}\left(i^{p} \sqrt[4]{z}\right) \\
{\left[1-\frac{1}{z} ;-2 p-2 q ; 2 p\right] } & =[z ; 2 p, 2 q]-\widehat{\chi}\left(e^{\left.-\frac{\pi i}{12}(1-6 p) \sqrt[4]{z}\right)}\right. \\
{\left[-\frac{z}{1-z} ; 2 p+2 q,-2 q\right] } & =-[z ; 2 p, 2 q]+\widehat{\chi}\left(e^{\left.-\frac{\pi i}{12}(1+6 q) \sqrt[4]{z-1}\right)}\right. \\
{\left[\frac{1}{1-z} ; 2 q,-2 p-2 q\right] } & =[z ; 2 p, 2 q]-\widehat{\chi}\left(e^{\left.-\frac{\pi i}{12}(2+6 q) \sqrt[4]{z-1}\right)},\right. \\
{[1-z ;-2 q,-2 p] } & =-[z ; 2 p, 2 q]+\widehat{\chi}\left(e^{\frac{\pi i}{12}}\right)
\end{aligned}
$$

Proof The involutions

$$
[z ; 2 p, 2 q] \mapsto[1-z ;-2 q,-2 p] \text { and }[z ; 2 p, 2 q] \mapsto\left[\frac{1}{z} ;-2 p ; 2 p+2 q\right]
$$

generate an action of $S(3)$ on $\widehat{\mathbb{C}}$. Using $\operatorname{Im}\left(1-\frac{1}{z}\right)>0$ and $\operatorname{Im} \frac{1}{1-z}>0$, it is now easy to see that the five relations above follow from (1) and (5). Note that by [5], both relations are true modulo $\operatorname{ker}\left(\widehat{\mathcal{P}}_{S L}(\mathbb{C}) \rightarrow \mathcal{P}(\mathbb{C})\right)=\operatorname{im} \widehat{\chi}$. Because the Rogers dilogarithm $\widehat{L}$ is injective on $\operatorname{im} \widehat{\chi}$ by (14), it suffices to check both relations after applying $\widehat{L}$. This can be done using some elementary facts about the classical dilogarithm, and is thus left to the reader.

5.2 Remark The relations in [8] look somewhat nicer, since the correction term does not involve the variable $z$. This is possible because in Neumann's definition of $\widehat{\mathcal{P}}_{P S L}(\mathbb{C})$, odd integers are allowed, so that one can consider the involution $[z ; p, q] \mapsto$ $[1 / z ;-p, 1+p+q]$.

Following [8], the various terms on the left hand side of the equations in Proposition 5.1 correspond to flattenings of a given oriented simplex with different orderings of vertices. 
Thus the proposition seems to indicate that it is not enough to consider unordered oriented simplices if one wants to compute the full class $\widehat{c}_{2}$.

For higher classes $\widehat{c}_{k}$, not many formulas are available. The only formula known to the authors is Goncharov's formula for Re $\widehat{c}_{3}$ in [7], which uses unordered oriented simplices.

\section{References}

[1] J Cheeger, J Simons, Differential characters and geometric invariants, from: "Geometry and topology (College Park, MD, 1983/84)", Lecture Notes in Mathematics 1167, Springer, Berlin (1985) 50-80 MR827262

[2] S S Chern, J Simons, Characteristic forms and geometric invariants, Ann. of Math. (2) 99 (1974) 48-69 MR0353327

[3] J L Dupont, The dilogarithm as a characteristic class for flat bundles, J. Pure Appl. Algebra 44 (1-3) (1987) 137-164 MR885101

[4] J L Dupont, Scissors congruences, group homology and characteristic classes, Nankai Tracts in Mathematics 1, World Scientific Publishing Co., River Edge, NJ (2001) MR1832859

[5] J L Dupont, C H Sah, Scissors congruences II, J. Pure Appl. Algebra 25 (1982) 159195 MR662760

[6] J L Dupont, C K Zickert, A dilogarithmic formula for the Cheeger-Chern-Simons class, Geom. Topol. 10 (2006) 1347-1372 MR2255500

[7] A B Goncharov, Geometry of configurations, polylogarithms, and motivic cohomology, Adv. Math. 114 (1995) 197-318 MR1348706

[8] W D Neumann, Extended Bloch group and the Cheeger-Chern-Simons class, Geom. Topol. 8 (2004) 413-474 MR2033484

Mathematisches Institut, Universität Freiburg,

Eckerstr. 1, 79104 Freiburg, Germany

Department of Mathematics, Columbia University,

New York NY 10027, USA

sebastian.goette@math.uni-freiburg.de, zickert@math.columbia.edu

Proposed: Robion Kirby

Received: 5 June 2007

Seconded: Shigeyuki Morita, Joan Birman

Revised: 11 July 2007 